\title{
INDECOMPOSABLE MODULES FOR THE DUAL IMMACULATE BASIS OF QUASI-SYMMETRIC FUNCTIONS
}

\author{
CHRIS BERG, NANTEL BERGERON, FRANCO SALIOLA, LUIS SERRANO, \\ AND MIKE ZABROCKI \\ (Communicated by Jim Haglund)
}

\begin{abstract}
We construct indecomposable modules for the 0-Hecke algebra whose characteristics are the dual immaculate basis of the quasi-symmetric functions.
\end{abstract}

\section{INTRODUCTION}

The algebra of symmetric functions Sym has an important basis formed by Schur functions, which appear throughout mathematics. For example, they appear as the representatives for the Schubert classes in the cohomology of the Grassmannian, as the characters for the irreducible representations of the symmetric group and the general linear group, or as an orthonormal basis for the space of symmetric functions, to name a few. The algebra NSym of non-commutative symmetric functions projects under the forgetful map onto Sym, which injects into the algebra QSym of quasi-symmetric functions. NSym and QSym are dual Hopf algebras.

In [BBSSZ, the authors developed a basis for NSym, which satisfied many of the combinatorial properties of Schur functions. This basis, called the immaculate basis $\left\{\mathfrak{S}_{\alpha}\right\}$, projects onto Schur functions under the forgetful map. When indexed by a partition, the corresponding projection of the immaculate function is precisely the Schur function of the given partition.

The dual basis $\left\{\mathfrak{S}_{\alpha}^{*}\right\}$ is a basis for QSym. The main goal of this paper is to express the dual immaculate functions as characters of some representations, in the same way that Schur functions are the characters of the irreducible representations of the symmetric group. We achieve this in Theorem 3.5, where we realize them as the characteristic of certain indecomposable representations of the 0 -Hecke algebra.

\section{Prerequisites}

2.1. The symmetric group. The symmetric group $S_{n}$ is the group generated by the set of $\left\{s_{1}, s_{2}, \ldots, s_{n-1}\right\}$ satisfying the following relations:

$$
\begin{aligned}
s_{i}^{2} & =1 ; \\
s_{i} s_{i+1} s_{i} & =s_{i+1} s_{i} s_{i+1} \\
s_{i} s_{j} & =s_{j} s_{i} \text { if }|i-j|>1 .
\end{aligned}
$$

Received by the editors May 21, 2014 and, in revised form, July 3, 2013.

2010 Mathematics Subject Classification. Primary 05E05, 05E10, 20C08; Secondary 14N15, $20 \mathrm{C} 30$. 
2.2. Compositions and combinatorics. A partition of a non-negative integer $n$ is a tuple $\lambda=\left[\lambda_{1}, \lambda_{2}, \ldots, \lambda_{m}\right]$ of positive integers satisfying $\lambda_{1} \geq \lambda_{2} \geq \cdots \geq \lambda_{m}$ which sum to $n$. If $\lambda$ is a partition of $n$, one writes $\lambda \vdash n$. (When needed, we will consider partitions with zeroes at the end, but they are equivalent to the underlying partition made of positive numbers.) Partitions are of particular importance in algebraic combinatorics, as they index a basis for the symmetric functions of degree $n, \mathrm{Sym}_{n}$, and the character ring for the representations of the symmetric group $S_{n}$, among others. These concepts are intimately connected; we assume the reader is well versed in this area (see for instance Sagan for background details).

A composition of a non-negative integer $n$ is a tuple $\alpha=\left[\alpha_{1}, \alpha_{2}, \ldots, \alpha_{m}\right]$ of positive integers which sum to $n$. If $\alpha$ is a composition of $n$, one writes $\alpha \models n$. The entries $\alpha_{i}$ of the composition are referred to as the parts of the composition. The size of the composition is the sum of the parts and will be denoted $|\alpha|$. The length of the composition is the number of parts and will be denoted $\ell(\alpha)$. Note that $|\alpha|=n$ and $\ell(\alpha)=m$.

Compositions of $n$ are in bijection with subsets of $\{1,2, \ldots, n-1\}$. We will follow the convention of identifying $\alpha=\left[\alpha_{1}, \alpha_{2}, \ldots, \alpha_{m}\right]$ with the subset $\mathcal{S}(\alpha)=$ $\left\{\alpha_{1}, \alpha_{1}+\alpha_{2}, \alpha_{1}+\alpha_{2}+\alpha_{3}, \ldots, \alpha_{1}+\alpha_{2}+\cdots+\alpha_{m-1}\right\}$.

If $\alpha$ and $\beta$ are both compositions of $n$, we say that $\alpha \leq \beta$ in refinement order if $\mathcal{S}(\beta) \subseteq \mathcal{S}(\alpha) . \quad$ For instance, $[1,1,2,1,3,2,1,4,2] \leq[4,4,2,7]$, since $\mathcal{S}([1,1,2,1,3,2,1,4,2])=\{1,2,4,5,8,10,11,15\}$ and $\mathcal{S}([4,4,2,7])=\{4,8,10\}$.

In this presentation, compositions will be represented as diagrams of left adjusted rows of cells. We will also use the matrix convention ('English' notation) that the first row of the diagram is at the top and the last row is at the bottom. For example, the composition $[4,1,3,1,6,2]$ is represented as

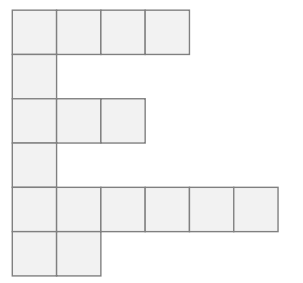

2.3. Symmetric functions. We let Sym denote the ring of symmetric functions. As an algebra, Sym is the ring over $\mathbb{Q}$ freely generated by commutative elements $\left\{h_{1}, h_{2}, \ldots\right\}$. Sym has a grading, defined by giving $h_{i}$ degree $i$ and extending multiplicatively. A natural basis for the degree $n$ component of Sym are the complete homogeneous symmetric functions of degree $n,\left\{h_{\lambda}:=h_{\lambda_{1}} h_{\lambda_{2}} \cdots h_{\lambda_{m}}: \lambda \vdash n\right\}$. It is common and advantageous to identify Sym with a subring of the ring of power series of bounded degree $\mathbb{Q} \llbracket x_{1}, x_{2}, \ldots \rrbracket$ in commuting variables $\left\{x_{1}, x_{2}, \ldots\right\}$; it consists of the elements that are invariant under any permutation of the variables. Under this identification, $h_{i}$ corresponds to the sum of all monomials of degree $i$.

2.4. Non-commutative symmetric functions. NSym is a non-commutative analogue of Sym, the algebra of symmetric functions, that arises by considering an algebra with one non-commutative generator at each positive degree. In addition to the relationship with the symmetric functions, this algebra has links to Solomon's descent algebra in type $A[\mathrm{MR}$, the algebra of quasi-symmetric functions $[\mathrm{MR}$, and representation theory of the type $A$ Hecke algebra at $q=0[\mathrm{KT}$. It is 
an example of a combinatorial Hopf algebra ABS. While we will follow the foundational results and definitions from references such as GKLLRT, MR, we have chosen to use notation here which is suggestive of analogous results in Sym.

We consider NSym as the algebra with generators $\left\{H_{1}, H_{2}, \ldots\right\}$ and no relations. Each generator $H_{i}$ is defined to be of degree $i$, giving NSym the structure of a graded algebra. We let $\mathrm{NSym}_{n}$ denote the graded component of NSym of degree $n$. A basis for $\mathrm{NSym}_{n}$ are the complete homogeneous functions $\left\{H_{\alpha}:=H_{\alpha_{1}} H_{\alpha_{2}} \cdots H_{\alpha_{m}}\right\}_{\alpha \models n}$ indexed by compositions of $n$.

\subsection{Immaculate tableaux.}

Definition 2.1. Let $\alpha$ and $\beta$ be compositions. An immaculate tableau of shape $\alpha$ and content $\beta$ is a labelling of the boxes of the diagram of $\alpha$ by positive integers in such a way that:

(1) the number of boxes labelled by $i$ is $\beta_{i}$;

(2) the sequence of entries in each row, from left to right, is weakly increasing;

(3) the sequence of entries in the first column, from top to bottom, is increasing.

An immaculate tableau is said to be standard if it has content $1^{|\alpha|}$.

Let $K_{\alpha, \beta}$ denote the number of immaculate tableaux of shape $\alpha$ and content $\beta$.

We reiterate that aside from the first column, there is no relation on the other columns of an immaculate tableau.

Example 2.2. The five immaculate tableaux of shape $[4,2,3]$ and content $[3,1,2,3]$ :

\begin{tabular}{|l|l|l|l|}
\hline 1 & 1 & 1 & 3 \\
\hline 2 & 3 & \multicolumn{3}{|c}{} \\
\cline { 1 - 2 } 4 & 4 & \multicolumn{2}{|l}{4} \\
\cline { 1 - 2 }
\end{tabular}

\begin{tabular}{|l|l|l|l|}
\hline 1 & 1 & 1 & 3 \\
\hline 2 & 4 & \multicolumn{3}{|c}{} \\
\cline { 1 - 2 } 3 & 4 & \multicolumn{3}{|l}{4} \\
\cline { 1 - 2 } &
\end{tabular}

\begin{tabular}{|l|l|l|l|}
\hline 1 & 1 & 1 & 4 \\
\hline 2 & 3 & \multicolumn{3}{|c}{} \\
\cline { 1 - 2 } 3 & 4 & \multicolumn{2}{|l}{4} \\
\cline { 1 - 2 }
\end{tabular}

\begin{tabular}{|l|l|l|l|}
\hline 1 & 1 & 1 & 4 \\
\hline 2 & 4 & \multicolumn{3}{|c}{} \\
\cline { 1 - 2 } 3 & 3 & 4 \\
\cline { 1 - 1 }
\end{tabular}

\begin{tabular}{|l|l|l|l|}
\hline 1 & 1 & 1 & 2 \\
\hline 3 & 3 & \multicolumn{2}{|c}{} \\
\cline { 1 - 2 } 4 & 4 & \multicolumn{2}{|l}{4} \\
\cline { 1 - 2 }
\end{tabular}

Definition 2.3. We say that a standard immaculate tableau $T$ has a descent in position $i$ if $i+1$ is in a row strictly below the row containing $i$. The descent composition, denoted $D(T)$, is the composition corresponding to the set of descents in $T$.

Example 2.4. The standard immaculate tableau of shape $[6,5,7]$ :

$$
T=\begin{array}{c|c|c|c|c|c|c}
\hline 1 & 2 & 4 & 5 & 10 & 11 \\
\hline 3 & 6 & 7 & 8 & 9 & \multicolumn{1}{|c}{} \\
\cline { 1 - 6 } 12 & 13 & 14 & 15 & 16 & 17 & 18 \\
\hline
\end{array}
$$

has descents in positions $\{2,5,11\}$. The descent composition of $T$ is then $D(T)=$ $[2,3,6,7]$.

2.6. The immaculate basis of NSym. The immaculate basis of NSym was introduced in BBSSZ]. It shares many properties with the Schur basis of Sym. We define 1 the immaculate basis $\left\{\mathfrak{S}_{\alpha}\right\}_{\alpha}$ as the unique elements of NSym satisfying:

$$
H_{\beta}=\sum_{\alpha} K_{\alpha, \beta} \mathfrak{S}_{\alpha} .
$$

Example 2.5. Continuing from Example 2.2, we see that

$$
H_{3123}=\cdots+5 \mathfrak{S}_{423}+\cdots .
$$

\footnotetext{
${ }^{1}$ This is not the original definition, but is equivalent by Proposition 3.16 in [BBSSZ.
} 
We will not attempt to summarize everything that is known about this basis, but instead refer the reader to [BBSSZ] and BBSSZ2].

\section{Modules for the DUAL immaculate Basis}

In this section we will construct indecomposable modules for the 0-Hecke algebra whose characteristic is a dual immaculate quasi-symmetric function.

3.1. Quasi-symmetric functions. The algebra of quasi-symmetric functions, QSym, was introduced in Ges] (see also subsequent references such as GR, Sta84]). The graded component QSym $_{n}$ is indexed by compositions of $n$. The algebra is most readily realized as a subalgebra of the ring of power series of bounded degree $\mathbb{Q} \llbracket x_{1}, x_{2}, \ldots \rrbracket$, and the monomial quasi-symmetric function indexed by a composition $\alpha$ is defined as

$$
M_{\alpha}=\sum_{i_{1}<i_{2}<\cdots<i_{m}} x_{i_{1}}^{\alpha_{1}} x_{i_{2}}^{\alpha_{2}} \cdots x_{i_{m}}^{\alpha_{m}}
$$

The algebra of quasi-symmetric functions, QSym, can be defined as the linear span of the monomial quasi-symmetric functions. These, in fact, form a basis of QSym, and their multiplication is inherited from $\mathbb{Q} \llbracket x_{1}, x_{2}, \ldots \rrbracket$. We view Sym as a subalgebra of QSym. In fact, the quasi-symmetric monomial functions refine the usual monomial symmetric functions $m_{\lambda} \in$ Sym:

$$
m_{\lambda}=\sum_{\operatorname{sort}(\alpha)=\lambda} M_{\alpha},
$$

where $\operatorname{sort}(\alpha)$ denotes the partition obtained by organizing the parts of $\alpha$ from the largest to the smallest.

The fundamental quasi-symmetric function, denoted $F_{\alpha}$, is defined by its expansion in the monomial quasi-symmetric basis:

$$
F_{\alpha}=\sum_{\beta \leq \alpha} M_{\beta}
$$

The algebras QSym and NSym form dual graded Hopf algebras. In this context, the monomial basis of QSym is dual to the complete homogeneous basis of NSym. Duality can be expressed by the means of an inner product, for which $\left\langle H_{\alpha}, M_{\beta}\right\rangle=$ $\delta_{\alpha, \beta}$.

In BBSSZ, we studied the dual basis to the immaculate functions of NSym, denoted $\mathfrak{S}_{\beta}^{*}$ and indexed by compositions. They are the basis of QSym defined by $\left\langle\mathfrak{S}_{\alpha}, \mathfrak{S}_{\beta}^{*}\right\rangle=\delta_{\alpha, \beta}$. In [BBSSZ, Proposition 3.37], we showed that the dual immaculate functions have the following positive expansion into the fundamental basis:

Proposition 3.1. The dual immaculate functions $\mathfrak{S}_{\alpha}^{*}$ are fundamental positive. Specifically they expand as

$$
\mathfrak{S}_{\alpha}^{*}=\sum_{T} F_{D(T)},
$$

a sum over all standard immaculate tableaux of shape $\alpha$. 
3.2. Finite dimensional representation theory of $H_{n}(0)$. We will outline the study of the finite dimensional representations of the 0-Hecke algebra and its relationship to QSym. We begin by defining the 0-Hecke algebra. We refer the reader to [Th2, Section 5] for the relationship between the generic Hecke algebra and the 0 -Hecke algebra and their connections to representation theory.

Definition 3.2. Let $\mathbb{K}$ be any field. The Hecke algebra $H_{n}(0)$ is the $\mathbb{K}$-algebra generated by the elements $\pi_{1}, \pi_{2}, \ldots \pi_{n-1}$ subject to relations:

$$
\begin{aligned}
\pi_{i}^{2} & =\pi_{i} \\
\pi_{i} \pi_{i+1} \pi_{i} & =\pi_{i+1} \pi_{i} \pi_{i+1} \\
\pi_{i} \pi_{j} & =\pi_{j} \pi_{i} \text { if }|i-j|>1 .
\end{aligned}
$$

A basis of $H_{n}(0)$ is given by the elements $\left\{\pi_{\sigma}: \sigma \in S_{n}\right\}$, where $\pi_{\sigma}=\pi_{i_{1}} \pi_{i_{2}} \cdots \pi_{i_{m}}$ if $\sigma=s_{i_{1}} s_{i_{2}} \cdots s_{i_{m}}$.

We let $G_{0}\left(H_{n}(0)\right)$ denote the Grothendieck group of finite dimensional representations of $H_{n}(0)$. As a vector space, $G_{0}\left(H_{n}(0)\right)$ is spanned by the finite dimensional representations of $H_{n}(0)$, with the relation on isomorphism classes $[B]=[A]+[C]$ whenever there is a short exact sequence of $H_{n}(0)$-representations $0 \rightarrow A \rightarrow B \rightarrow C \rightarrow 0$. We let

$$
\mathcal{G}=\bigoplus_{n \geq 0} G_{0}\left(H_{n}(0)\right)
$$

The irreducible representations of $H_{n}(0)$ are indexed by compositions of $n$. The irreducible representation corresponding to the composition $\alpha$ is denoted $L_{\alpha}$. The collection $\left\{\left[L_{\alpha}\right]\right\}$ forms a basis for $\mathcal{G}$. As shown in Norton $[\mathrm{N}$, each irreducible representation is one dimensional, spanned by a non-zero vector $v_{\alpha} \in L_{\alpha}$, and is determined by the action of the generators on $v_{\alpha}$ :

$$
\pi_{i} v_{\alpha}= \begin{cases}0 & \text { if } i \in \mathcal{S}(\alpha) \\ v_{\alpha} & \text { otherwise }\end{cases}
$$

where $\mathcal{S}(\alpha)$ denotes the subset of $[1 \ldots n-1]$ corresponding to the composition $\alpha$. The tensor product $H_{n}(0) \otimes H_{m}(0)$ is naturally embedded as a subalgebra of $H_{n+m}(0)$. Under this identification, one can endow $\mathcal{G}$ with a ring structure; for $[N] \in G_{0}\left(H_{n}(0)\right)$ and $[M] \in G_{0}\left(H_{m}(0)\right)$, let

$$
[N][M]:=\left[\operatorname{Ind}_{H_{n}(0) \otimes H_{m}(0)}^{H_{n+m}(0)} N \otimes M\right]
$$

where induction is defined in the usual manner.

There is an important linear map $\mathcal{F}: \mathcal{G} \rightarrow$ QSym defined by $\mathcal{F}\left(\left[L_{\alpha}\right]\right)=F_{\alpha}$. For a module $M, \mathcal{F}([M])$ is called the characteristic of $M$.

Theorem 3.3 (Duchamp, Krob, Leclerc, Thibon [DKLT]). The quasi-symmetric functions and the Grothendieck group of finite dimensional representations of $H_{n}(0)$ are isomorphic as rings. The map $\mathcal{F}$ is an isomorphism between $\mathcal{G}$ and QSym.

Remark 3.4. The map $\mathcal{F}$ is actually an isomorphism of graded Hopf algebras. We will not make use of the coalgebra structure. 
3.3. A representation on $\mathcal{Y}$-words. We start by defining the analogue of a permutation module for $H_{n}(0)$. For a composition $\alpha=\left[\alpha_{1}, \alpha_{2}, \ldots, \alpha_{m}\right] \models n$, we let $\mathcal{M}_{\alpha}$ denote the vector space spanned by words of length $n$ on the letters $\{1,2, \ldots, m\}$ with content $\alpha$ (so that $j$ appears $\alpha_{j}$ times in each word). The action of $H_{n}(0)$ on a word $w=w_{1} w_{2} \cdots w_{n}$ is defined on generators as:

$$
\pi_{i} w= \begin{cases}w & \text { if } w_{i} \geq w_{i+1} \\ s_{i}(w) & \text { if } w_{i}<w_{i+1}\end{cases}
$$

where $s_{i}(w)=w_{1} w_{2} \cdots w_{i+1} w_{i} \cdots w_{n}$. This is isomorphic to the representation:

$$
\operatorname{Ind}_{H_{\alpha}(0)}^{H_{n}(0)}\left(L_{\alpha_{1}} \otimes L_{\alpha_{2}} \otimes \cdots \otimes L_{\alpha_{m}}\right),
$$

where $L_{k}$ is the one-dimensional representation indexed by the composition $[k]$ and $H_{\alpha}(0):=H_{\alpha_{1}}(0) \otimes H_{\alpha_{2}}(0) \otimes \cdots \otimes H_{\alpha_{m}}(0)$. This can be seen by associating the element $\pi_{v} \otimes_{H_{\alpha}(0)} L_{\alpha_{1}} \otimes L_{\alpha_{2}} \otimes \cdots \otimes L_{\alpha_{m}}$ where $v$ is the minimal length left coset representative of $S_{n} / S_{\alpha_{1}} \times S_{\alpha_{2}} \times \cdots \times S_{\alpha_{m}}$ with the element $\pi_{v}\left(1^{\alpha_{1}} 2^{\alpha_{2}} \cdots k^{\alpha_{k}}\right)$.

We call a word a $\mathcal{Y}$-word if the first instance of $j$ appears before the first instance of $j+1$ for every $j$. We let $\mathcal{N}_{\alpha}$ denote the subspace of $\mathcal{M}_{\alpha}$ spanned by all words that are not $\mathcal{Y}$-words. The action of $H_{n}(0)$ on $\mathcal{M}_{\alpha}$ will never move a $j+1$ to the right of a $j$. This implies that $\mathcal{N}_{\alpha}$ is a submodule of $\mathcal{M}_{\alpha}$. The object of our interest is the quotient module $\mathcal{V}_{\alpha}:=\mathcal{M}_{\alpha} / \mathcal{N}_{\alpha}$. We now state our main result.

Theorem 3.5. The characteristic of $\mathcal{V}_{\alpha}$ is the dual immaculate function indexed by $\alpha$, i.e. $\mathcal{F}\left(\left[\mathcal{V}_{\alpha}\right]\right)=\mathfrak{S}_{\alpha}^{*}$.

Before we prove this we will associate words to standard immaculate tableaux and give an equivalent description of the 0-Hecke algebra on standard immaculate tableau. To a $\mathcal{Y}$-word $w$, we associate the unique standard immaculate tableau $\mathcal{T}(w)$ which has a $j$ in row $w_{j}$.

Example 3.6. Let $w=112322231$ be the $\mathcal{Y}$-word of content $[3,4,2]$. Then $\mathcal{T}(w)$ is the standard immaculate tableau:

$$
\begin{array}{|l|l|l|l|}
\hline 1 & 2 & 9 & \multicolumn{1}{|}{} \\
\hline 3 & 5 & 6 & 7 \\
\cline { 1 - 2 } 4 & 8 & \multicolumn{2}{|c}{} \\
\cline { 1 - 2 } & & &
\end{array}
$$

Remark 3.7. $\mathcal{T}$ yields a bijection between standard immaculate tableaux and $\mathcal{Y}$ words.

Remark 3.8. In the case of the symmetric group, the irreducible module corresponding to the partition $\lambda$ has a basis indexed by standard tableaux. Under the same map $\mathcal{T}$, standard Young tableaux are in bijection with Yamanouchi words (words for which every prefix contains at least as many $j$ as $j+1$ for every $j$ ). In this sense, $\mathcal{Y}$-words are a natural analogue to Yamanouchi words in our setting. The Specht modules that give rise to the irreducible modules of the symmetric group are built as quotients of $\mathcal{M}_{\lambda}$. Under the Frobenius map, these modules are sent to Schur functions if the field has characteristic 0 . 
We may now describe the action of $H_{n}(0)$ on $\mathcal{V}_{\alpha}$, identifying the set of standard immaculate tableaux as the basis. Specifically, for a tableau $T$ and a generator $\pi_{i}$, we let:

$$
\pi_{i}(T)= \begin{cases}0 & \text { if } i \text { and } i+1 \text { are in the first column of } T \\ T & \text { if } i \text { is in a row weakly below the row containing } i+1 \\ s_{i}(T) & \text { otherwise; }\end{cases}
$$

where $s_{i}(T)$ is the tableau that differs from $T$ by swapping the letters $i$ and $i+1$.

Example 3.9. Continuing from Example 3.6, we see that $\pi_{1}, \pi_{4}, \pi_{5}, \pi_{6}, \pi_{8}$ send $T$ to itself, $\pi_{3}$ sends $T$ to 0 and $\pi_{2}, \pi_{7}$ send $T$ to the following tableaux:

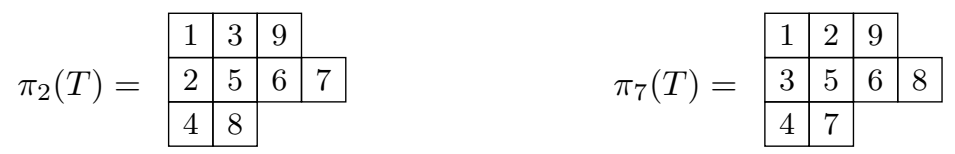

An example of the full action of $\pi_{i}$ on tableaux representing the basis elements of the module $\mathcal{V}_{(2,2,3)}$ is given in Figure 1 If we order the tableaux so that $S \prec T$ if there exists a permutation $\sigma$ such that $\pi_{\sigma}(T)=S$, then this figure shows that order is not a total order on tableaux but that it can be extended to a total order arbitrarily. We will use this total order in the following proof of Theorem 3.5.

We are now ready to prove Theorem 3.5. which states that the characteristic of $\mathcal{V}_{\alpha}$ is $\mathfrak{S}_{\alpha}^{*}$.

Proof of Theorem 3.5. We construct a filtration of the module $\mathcal{V}_{\alpha}$ whose successive quotients are irreducible representations. Now, define $\mathcal{M}_{T}$ to be the linear span of all standard immaculate tableaux that are less than or equal to $T$. From the definition of the order and the fact that the $\pi_{i}$ are not invertible, we see that $\mathcal{M}_{T}$ is a module. Ordering the standard immaculate tableaux of shape $\alpha$ as $T_{1}, T_{2}, \ldots, T_{m}$, then we have a filtration of $\mathcal{V}_{\alpha}$ :

$$
0 \subset \mathcal{M}_{T_{1}} \subset \mathcal{M}_{T_{2}} \subset \cdots \subset \mathcal{M}_{T_{m}}=\mathcal{V}_{\alpha} .
$$

The successive quotient modules $\mathcal{M}_{T_{j}} / \mathcal{M}_{T_{j-1}}$ are one dimensional, spanned by $T_{j}$; to determine which irreducible this is, it suffices to compute the action of the generators. From the description of $\mathcal{V}_{\alpha}$ above, we see that

$$
\pi_{i}\left(T_{j}\right)= \begin{cases}0 & \text { if } i \in \mathcal{S}\left(D\left(T_{j}\right)\right) \\ T_{j} & \text { otherwise. }\end{cases}
$$

This is the representation $\left[L_{D\left(T_{j}\right)}\right]$, whose characteristic is $F_{D\left(T_{j}\right)}$. Therefore $\mathcal{F}\left(\left[\mathcal{V}_{\alpha}\right]\right)=\mathfrak{S}_{\alpha}^{*}$ by Proposition 3.1 ,

We aim to prove that the modules we have constructed are indecomposable. We let $\hat{S}_{\alpha}$ denote the super-standard tableau of shape $\alpha$, namely, the unique standard immaculate tableau with the first $\alpha_{1}$ letters in the first row, the next $\alpha_{2}$ letters in the second row, etc. We will first need a few lemmas.

Lemma 3.10. The module $\mathcal{V}_{\alpha}$ is cyclicly generated by $\hat{S}_{\alpha}$.

Proof. The module $\mathcal{M}_{\alpha}$ is cyclically generated by $1^{\alpha_{1}} 2^{\alpha_{2}} \cdots k^{\alpha_{k}}=\mathcal{T}^{-1}\left(\hat{S}_{\alpha}\right)$, which can be seen since every basis element of $\mathcal{M}_{\alpha}$ comes from an application of the anti-sorting operators $\pi_{i}$ on $1^{\alpha_{1}} 2^{\alpha_{2}} \cdots k^{\alpha_{k}}$.

$\mathcal{V}_{\alpha}$ is a quotient of $\mathcal{M}_{\alpha}$, and hence cyclicly generated by the same element. 


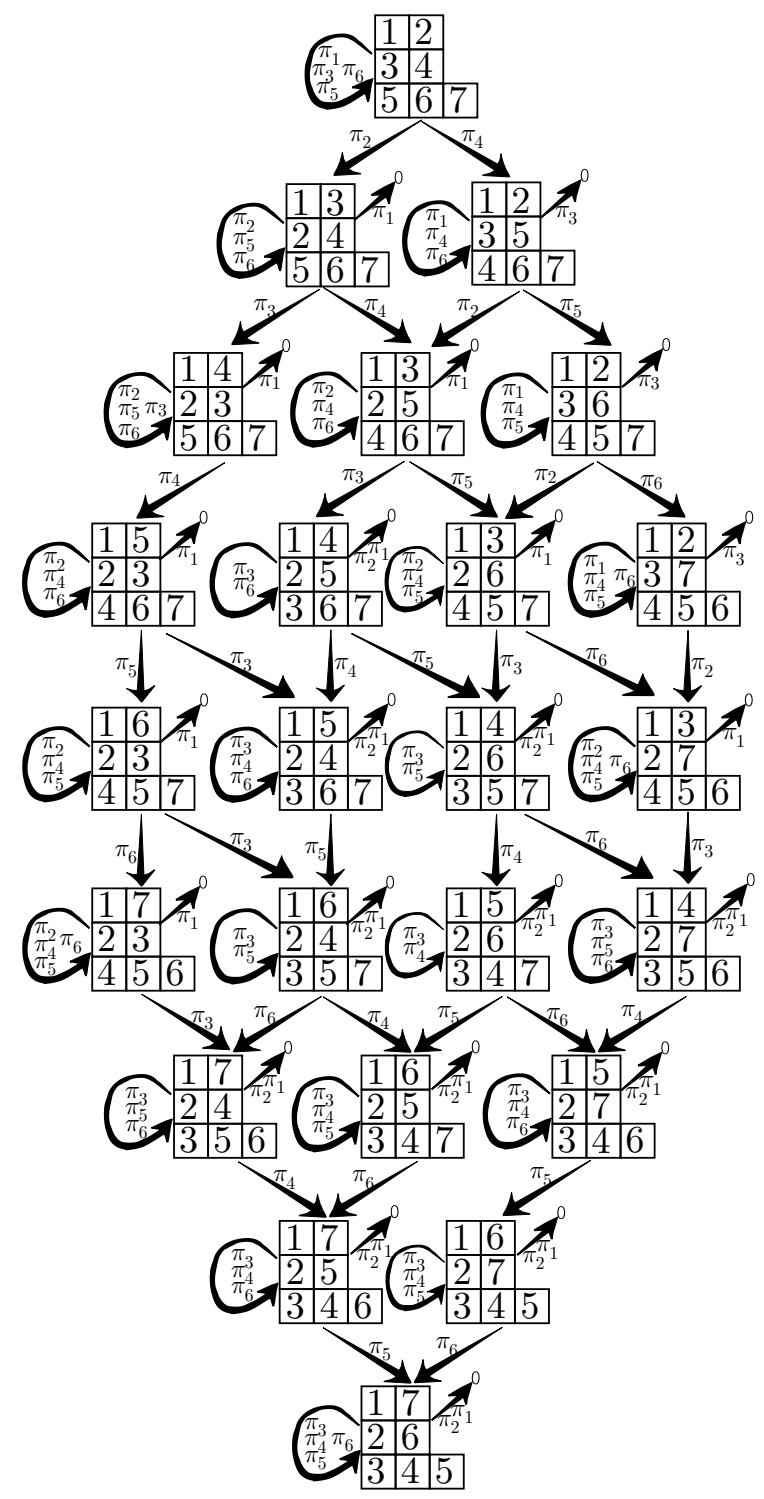

FiguRE 1. A diagram representing the action of the generators $\pi_{i}$ of $H_{n}(0)$ given in equation (3.4) on the basis elements of the module $\mathcal{V}_{(2,2,3)}$.

Lemma 3.11. If $P$ is a standard immaculate tableau of shape $\alpha$ such that $\pi_{i}(P)=$ $P$ for all $i \in\{1,2, \cdots, n\} \backslash \mathcal{S}(\alpha)$, then $P=\hat{S}_{\alpha}$. In particular, if $P \neq \hat{S}_{\alpha}$, then there exists an $i$ such that $\pi_{i}\left(\hat{S}_{\alpha}\right)=\hat{S}_{\alpha}$ but $\pi_{i}(P) \neq P$.

Proof. If $\pi_{i}(P)=P$, then $i$ must be in the cell to the left of $i+1$ or in a row below $i+1$. The fact that $\pi_{i}(P)=P$ for all $i \in\left\{1,2, \ldots, \alpha_{1}-1\right\}$ implies that the first 
row of $P$ agrees with $\hat{S}_{\alpha}$. In a similar manner, we see that the second rows must agree. Continuing in this manner, we conclude that $P=\hat{S}_{\alpha}$.

Theorem 3.12. For every $\alpha \models n, \mathcal{V}_{\alpha}$ is an indecomposable representation of $H_{n}(0)$.

Proof. We let $f$ be an idempotent module morphism from $\mathcal{V}_{\alpha}$ to itself. If we can prove $f$ is either the zero morphism or the identity, then $\mathcal{V}_{\alpha}$ is indecomposable [Ja, Proposition 3.1].

Suppose $f\left(\hat{S}_{\alpha}\right)=\sum_{T} a_{T} T$. By Lemma 3.11, for any $P \neq \hat{S}_{\alpha}$, there exists an $i$ such that $\pi_{i}\left(\hat{S}_{\alpha}\right)=\hat{S}_{\alpha}$ but $\pi_{i}(P) \neq P$. Since $f$ is a module map,

$$
\sum_{T} a_{T} T=f\left(\hat{S}_{\alpha}\right)=f\left(\pi_{i} \hat{S}_{\alpha}\right)=\pi_{i} f\left(\hat{S}_{\alpha}\right)=\sum_{T} a_{T} \pi_{i} T .
$$

The coefficient of $P$ on the right-hand side of equation (3.6) is zero (if there was a $T$ such that $\pi_{i} T=P$, then $\pi_{i} T=\pi_{i}^{2} T=\pi_{i} P \neq P$, a contradiction). Therefore $a_{P}=0$ for all $P \neq \hat{S}_{\alpha}$, so $f\left(\hat{S}_{\alpha}\right)=\hat{S}_{\alpha}$, or $f\left(\hat{S}_{\alpha}\right)=0$. Since $\mathcal{V}_{\alpha}$ is cyclicly generated by $\hat{S}_{\alpha}$, this implies that either $f$ is the identity morphism or the zero morphism.

Remark 3.13. In general, $\mathcal{V}_{\alpha}$ is not a projective $H_{n}(0)$-module. This is because the Frobenius characteristic of a projective indecomposable $H_{n}(0)$-module is a symmetric function [DKLT,, $\mathrm{KT}$, whereas the Frobenius characteristic of $\mathcal{V}_{\alpha}$ is the dual immaculate function indexed by $\alpha$ and hence is a quasi-symmetric function (see Theorem 3.5. .

\section{ACKNOWLEDGEMENTS}

This work was supported in part by NSERC and FRQNT. It is partially the result of a working session at the Algebraic Combinatorics Seminar at the Fields Institute with the active participation of C. Benedetti, J. Sánchez-Ortega, O. Yacobi, E. Ens, H. Heglin, D. Mazur and T. MacHenry.

This research was facilitated by computer exploration using the open-source mathematical software Sage [sage] and its algebraic combinatorics features developed by the Sage-Combinat community sage-combinat.

The authors would like to thank the anonymous referee for valuable comments on the exposition.

\section{REFERENCES}

[ABS] Marcelo Aguiar, Nantel Bergeron, and Frank Sottile, Combinatorial Hopf algebras and generalized Dehn-Sommerville relations, Compos. Math. 142 (2006), no. 1, 1-30, DOI 10.1112/S0010437X0500165X. MR2196760(2006h:05233)

[BBSSZ] C. Berg, N. Bergeron, F. Saliola, L. Serrano and M. Zabrocki, A lift of the Schur and Hall-Littlewood bases to non-commutative symmetric functions, arXiv:1208.5191, to appear, Canadian Journal of Mathematics, doi:10.4153/CJM2013-013-0.

[BBSSZ2] C. Berg, N. Bergeron, F. Saliola, L. Serrano and M. Zabrocki, Multiplicative structures of the immaculate basis of non-commutative symmetric functions, arXiv:1305.4700.

[DKLT] Gérard Duchamp, Daniel Krob, Bernard Leclerc, and Jean-Yves Thibon, Fonctions quasi-symétriques, fonctions symétriques non commutatives et algèbres de Hecke à $q=0$ (French, with English and French summaries), C. R. Acad. Sci. Paris Sér. I Math. 322 (1996), no. 2, 107-112. MR1373744(97a:05211) 
[Ges] Ira M. Gessel, Multipartite P-partitions and inner products of skew Schur functions, Combinatorics and algebra (Boulder, Colo., 1983), Contemp. Math., vol. 34, Amer. Math. Soc., Providence, RI, 1984, pp. 289-317, DOI 10.1090/conm/034/777705. MR7777705 (86k:05007)

[GKLLRT] Israel M. Gelfand, Daniel Krob, Alain Lascoux, Bernard Leclerc, Vladimir S. Retakh, and Jean-Yves Thibon, Noncommutative symmetric functions, Adv. Math. 112 (1995), no. 2, 218-348, DOI 10.1006/aima.1995.1032. MR1327096 (96e:05175)

[GR] Ira M. Gessel and Christophe Reutenauer, Counting permutations with given cycle structure and descent set, J. Combin. Theory Ser. A 64 (1993), no. 2, 189215, DOI 10.1016/0097-3165(93)90095-P. MR1245159(95g:05006)

[Ja] N. Jacobson, Basic algebra 2, (2nd ed.), Dover.

[KT] Daniel Krob and Jean-Yves Thibon, Noncommutative symmetric functions. IV. Quantum linear groups and Hecke algebras at $q=0$, J. Algebraic Combin. 6 (1997), no. 4, 339-376, DOI 10.1023/A:1008673127310. MR:1471894 (99c:05196)

[MR] Clauda Malvenuto and Christophe Reutenauer, Duality between quasi-symmetric functions and the Solomon descent algebra, J. Algebra 177 (1995), no. 3, 967-982, DOI 10.1006/jabr.1995.1336. MR1358493 (97d:05277)

[N] P. N. Norton, 0-Hecke algebras, J. Austral. Math. Soc. Ser. A 27 (1979), no. 3, 337-357. MR532754 (80e:16015)

[Sagan] Bruce E. Sagan, The symmetric group: Representations, combinatorial algorithms, and symmetric functions, 2nd ed., Graduate Texts in Mathematics, vol. 203, Springer-Verlag, New York, 2001. MR.1824028 (2001m:05261)

[sage] W. A. Stein et al. Sage Mathematics Software (Version 4.3.3), The Sage Development Team, 2010, http://www.sagemath.org.

[sage-combinat] The Sage-Combinat community. Sage-Combinat: enhancing Sage as a toolbox for computer exploration in algebraic combinatorics, http://combinat. sagemath.org, 2008.

[Sta84] Richard P. Stanley, On the number of reduced decompositions of elements of Coxeter groups, European J. Combin. 5 (1984), no. 4, 359-372. MR782057 (86i:05011)

[Th] Jean-Yves Thibon, Lectures on noncommutative symmetric functions, Interaction of combinatorics and representation theory, MSJ Mem., vol. 11, Math. Soc. Japan, Tokyo, 2001, pp. 39-94. MR1862149 (2003e:05142)

[Th2] J. Y. Thibon, Introduction to noncommutative symmetric functions, From Numbers and Languages to (Quantum) Cryptography, NATO Security through Science Series: Information and Communication Security, Volume 7.

Department of Mathematics, Université du Québec À Montréal, Montréal, Quebec H3C 3P8, CANADA

E-mail address: cberg@lacim.ca

Fields Institute, Toronto, Ontario M5T 3J1, Canada

Current address: Department of Mathematics and Statistics, York University, 4700 Keele Street, Toronto, Ontario M3J 1P3, Canada

E-mail address: bergeron@yorku.ca

Department of Mathematics, Université du Québec à Montréal, Montréal, Quebec H3C 3P8, CANADA

E-mail address: saliola@gmail.com

Department of Mathematics, Université du Québec à Montréal, Montréal, Quebec H3C 3P8, CANADA

E-mail address: serrano@lacim.ca

Fields Institute, Toronto, Ontario M5T 3J1, Canada

E-mail address: zabrocki@mathstat.yorku.ca 